\title{
Monte Carlo simulation approach for a quantitative characterization of the band edge in InGaN quantum wells
}

\author{
K. Kazlauskas ${ }^{* 1}$, G. Tamulatis ${ }^{1}$, S. Juršènas ${ }^{1}$, A. Žukauskas ${ }^{1}$, M. Springis ${ }^{2}$, \\ Yung-Chen Cheng ${ }^{3}$, Hsiang-Chen Wang ${ }^{3}$, Chi-Feng Huang ${ }^{3}$, and C. C. Yang ${ }^{3}$ \\ ${ }^{1}$ Institute of Materials Science and Applied Research, Vilnius University, \\ Sauletekio 9, Build. III, 10222 Vilnius, Lithuania \\ 2 Institute of Solid State Physics, University of Latvia, Kengaraga iela 8, 1063 Riga, Latvia \\ ${ }^{3}$ Graduate Institute of Electro-Optical Engineering, National Taiwan University, \\ 1 Roosevelt Road, Sec. 4, Taipei, Taiwan
}

Received 7 September 2004, accepted 22 September 2004

Published online 18 February 2005

PACS 02.50.Ng, 71.23.An, 78.40.Fy, 78.55.Cr, 78.67.De

\begin{abstract}
Monte Carlo simulation approach based on exciton hopping through randomly distributed localized states is proposed for quantitative characterization of the band edge of $\operatorname{In}_{x} \mathrm{Ga}_{1-x} \mathrm{~N} / \mathrm{GaN}$ multiple quantum wells with different indium content $(x \approx 0.22-0.27)$. The band edge dynamics is investigated in the $10-300 \mathrm{~K}$ range by analyzing the measured $\mathrm{S}$ - and $\mathrm{W}$-shaped temperature behavior of the photoluminescence peak position and linewidth, respectively. The simulation of the $\mathrm{W}$-shaped temperature dependence using double-scaled potential profile model enabled us to estimate the scale of the potential fluctuations due to variation of indium content inside and among In-rich regions formed in InGaN alloy. Increased indium content in InGaN alloy resulted in an increase of the both scales of the potential fluctuations. Moreover, the temperature dependence of the exciton energy was reconstructed and compared with that obtained from the photoreflectance measurements. The density of localized states used in the simulations was in agreement with the photoluminescence excitation data.
\end{abstract}

(C) 2005 WILEY-VCH Verlag GmbH \& Co. KGaA, Weinheim

1 Introduction InGaN is the key compound of the state-of-the-art blue-UV light emitting diodes and laser diodes. InGaN layers have inherent compositional fluctuations resulting in potential fluctuations, which localize carriers/excitons so that their nonradiative recombination is inhibited and the light emission efficiency is increased [1]. Fluctuations of alloy composition, i.e. formation of In-rich regions, have been observed in spatially resolved cathodoluminescence [2], near field optical microscopy [3], high resolution transmission electron microscopy, and X-ray diffraction microanalysis $[4,5]$ experiments. Recently, the existence of the In-rich regions resulting in "anomalous" temperature behavior of the PL peak and linewidth has been quantitatively explained by Monte Carlo simulation of exciton hopping [6]. However, the inherent disorder caused by inhomogeneous distribution of indium obscures the band edge of InGaN, which is a fundamental optical property.

In this report we exploit photoluminescence (PL), PL excitation, and photoreflectance spectroscopy to characterize the band edge of InGaN multiple quantum wells with different In content (22-27\%) and apply Monte Carlo simulation of exciton hopping to quantitatively describe the band edge dynamics in the temperature range of 10-300 $\mathrm{K}$ and to estimate the scales of the potential fluctuations in the alloy.

2 Experimental Three $\mathrm{In}_{x} \mathrm{Ga}_{1-x} \mathrm{~N} / \mathrm{GaN}$ multiple quantum well (MQW) samples with different indium content $(x \approx 0.22,0.25,0.27)$ were investigated. The samples consisted of five 2.5 -nm thick $\operatorname{In}_{x} \mathrm{Ga}_{1-x} \mathrm{~N}$

"Corresponding author: e-mail: karolis.kazlauskas@ff.vu.It, Phone: +370 5236 6096, Fax: +370 52366059 
quantum wells separated by $9-\mathrm{nm}$ thick GaN barriers deposited on GaN/sapphire templates using MOCVD technique.

A continuous-wave and chopped emission of a $15 \mathrm{~mW} \mathrm{He}-\mathrm{Cd}$ laser (325 nm) was used for excitation in PL and PR experiments, respectively. The PLE spectra were measured by using a 150-W Xe lamp. The optical signal was dispersed by a double monochromator and recorded by a photomultiplier. PL was measured in the photon-counting regime. Distortions of the PL line shape due to Fabry-Perrot interference were avoided by collecting the PL signal through the frosted transparent substrate. In the PR measurements, the modulated constituent of the reflected radiation from a 55-W halogen lamp was detected by means of a lock-in amplifier. The measurements in the $10-310 \mathrm{~K}$ temperature range were carried out by using a closed-cycle helium refrigerator.

3 Results and discussion Figure 1 shows the temperature evolution of the near-band-edge PL spectra of three InGaN MQW samples with different content of indium (22-27\%), measured in the range of 10-310 K. The PL band peak positions exhibit a well-established S-shaped temperature behaviour, whereas W-shaped dependences are observed for the full width at half maximum (FWHM) of the PL bands (shown by points in Fig. 2 (a)). The S- and W-shaped dependences are known to be a signature of exciton hopping through randomly dispersed localized states with a crossover from a nonthermalized to a thermalized distribution function of the excitons [6]. As a result of localization in the alloy, the PL bands are Stokes shifted with respect to the exciton energy. This Stokes shift scales with an amount of disorder and makes the quantitative estimation of the band edge in the alloy difficult.

The PLE spectra of all three samples at $77 \mathrm{~K}$ are displayed in Fig. 3 by bold lines. The spectra exhibit flat slopes in the long-wavelength spectral region, below the band gap of $\mathrm{GaN}(\sim 3.5 \mathrm{eV})$, corresponding to the absorption region of InGaN QWs. The temperature dependences of these PLE spectra, measured in the range of 10-300 K, revealed almost no difference in their slopes, what implies a significant contribu-

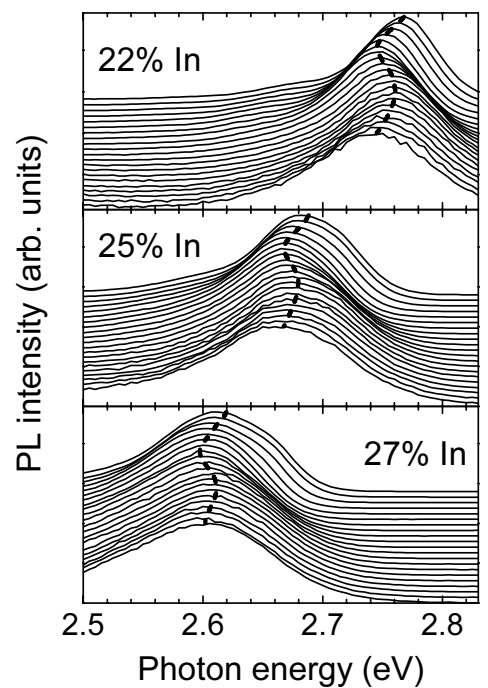

Fig. 1 Temperature evolution of the PL spectra in InGaN MQWs with $22 \%, 25 \%$ and $27 \%$ of indium. The temperature is incremented by $15 \mathrm{~K}$ starting with $10 \mathrm{~K}$ (the uppermost spectrum) and ending with $310 \mathrm{~K}$ (the lowermost spectrum). The spectra are normalized and arbitrarily shifted in a vertical direction for clarity. The band peak positions are highlighted by dashed lines.

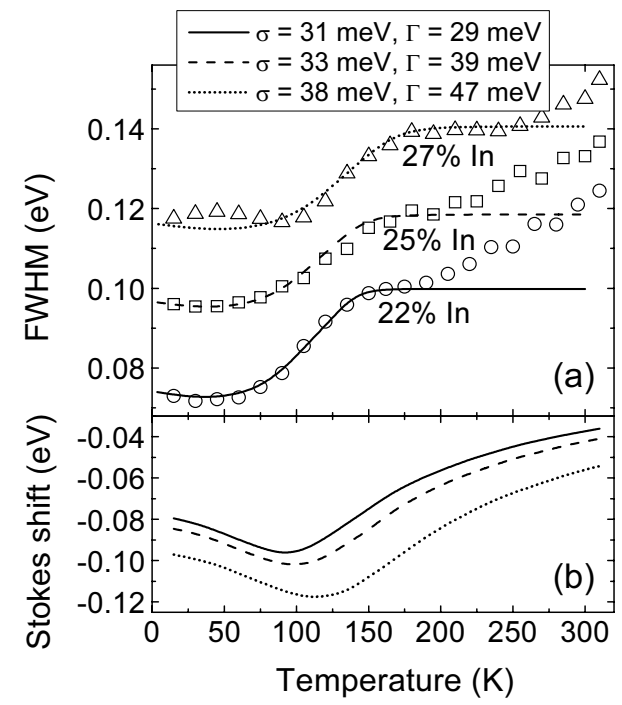

Fig. 2 (a) Experimental (points) and simulated (lines) temperature dependences of the PL linewidth in InGaN QWs with 22\% (circles and solid line), 25\% (rectangles and dashed line), and 27\% (triangles and dotted line) of indium. The dispersion parameter of the potential fluctuations $\sigma$ and inhomogeneous broadening $\Gamma$ are indicated. (b) Temperature dependence of the simulated Stokes shift of the PL band peak position in respect of the average exciton energy corresponding to the best-fitted linewidth dependence. 
tion of disorder due to the compositional fluctuations and interface roughness in respect to thermal broadening. Measurements of the reflectance spectra of the samples under investigation are also not helpful in quantifying the band edge because the spectra contain pronounced interference fringes in the vicinity of the band edge. Hence, neither PL nor PLE nor reflectance spectra are suitable for quantitative analysis of the band edge in partially disordered InGaN/GaN MQWs.

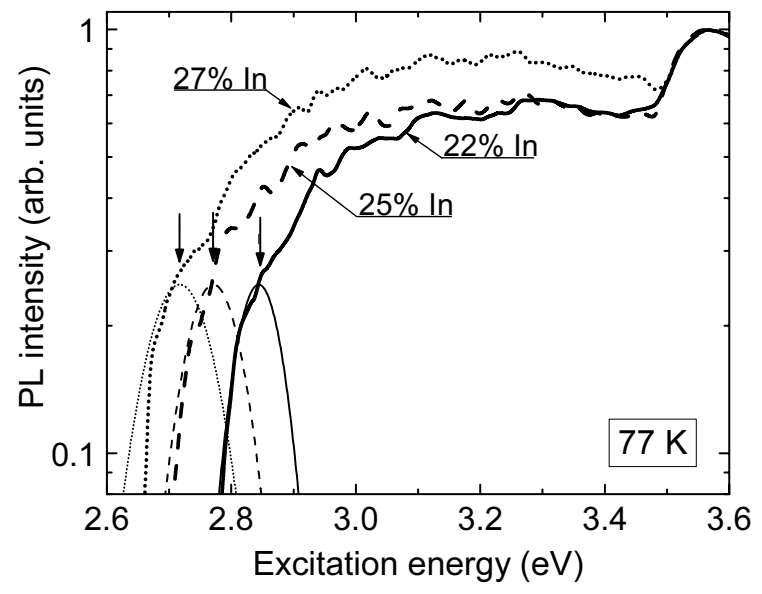

Fig. 3 PLE spectra of $2.5 \mathrm{~nm}$-thick InGaN/GaN MQWs with different In content, measured at $77 \mathrm{~K}$ (bold lines). Thin lines show the inhomogeneously broadened density of band-tail-states used in simulation. Vertical arrows indicate the exciton energy obtained from the simulation.

A 2D Monte Carlo simulation with the Miller-Abrahams rate for phonon-assisted exciton tunnelling between the localized states was applied to quantitatively describe the measured temperature dependences of the PL linewidths of InGaN MQWs and to extract the band edge parameters. The simulation procedure is described in more details in Refs. [6, 7]. Lines in Fig. 2 (a) illustrate the simulation results obtained using a double-scaled potential profile model. We stress that only after the introducing of this model we were able to quantitatively describe the PL linewidths. This model assumes exciton hopping through the potential fluctuations (with the dispersion parameter $\sigma$ ) in the individual In-rich regions with different average In content resulting in dispersion of the average exciton energy (on the energy scale $\Gamma$ ). In the case of QWs, $\Gamma$ may contain an additional contribution due to fluctuations in size of the In-rich regions and local built-in field strength. Obviously, an increase of indium content (from $22 \%$ to $27 \%$ ) in InGaN MQWs results in an increase of $\sigma$ (from 31 to $38 \mathrm{meV}$ ) as well as of $\Gamma$ (from 29 to $47 \mathrm{meV}$ ). A deviation of simulated linewidths from the experimental ones at the highest temperatures is due to the delocalized states, which were not taken into account.

Figure 2 (b) depicts the temperature behavior of the Stokes shift in InGaN QWs with different In content deduced from the peak position of the simulated spectra. Based on this data and the measured peak positions of the PL bands, the temperature dependences of the average exciton energy in the QWs with different In content were reconstructed (filled points in Fig. 5). The dependences are fairly well described by a Bose-Einstein-like expression (solid lines in Fig. 5) [8]

$$
E(T)=E(0)-\frac{\lambda}{\exp (\theta / T)-1}
$$

with the best-fit parameters: $\lambda=0.154 \mathrm{eV}, \theta=379 \mathrm{~K}, E(0)=2.845 \mathrm{eV}$ (for QWs with $22 \% \mathrm{In}$ ); $\lambda=$ $0.154 \mathrm{eV}, \theta=374 \mathrm{~K}, E(0)=2.773 \mathrm{eV}$ (for QWs with $25 \% \mathrm{In}$ ); $\lambda=0.274 \mathrm{eV}, \theta=520 \mathrm{~K}, E(0)=2.717 \mathrm{eV}$ (for QWs with 27\% In). The reconstructed dependences for each InGaN/GaN sample were collated with their respective energies of the PR resonances shown in Fig. 4. Despite a large uncertainty of the PR data caused by the broad PR features, a fair agreement with the data extracted using Monte Carlo simulation is obtained. This suggests that at lower temperatures, where PR can be difficult to measure, the simulation provides with unique data on the fundamental optical transition energy. Although obtained in a rather indirect way, this data can be useful for characterization of thin quantum wells with relatively high In molar fractions, where the exciton energy is difficult to precisely measure by other means. 


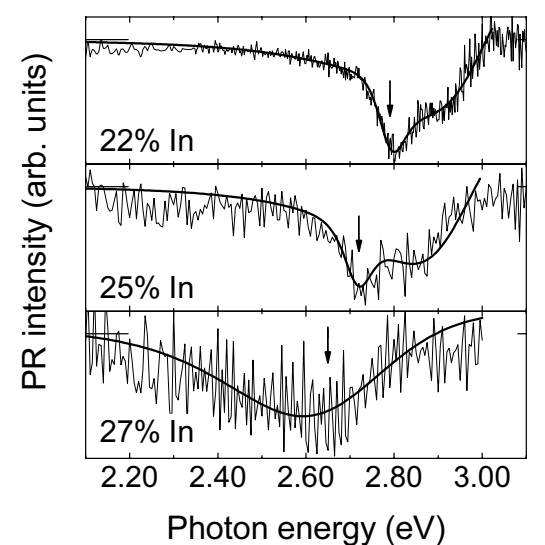

Fig. 4 Room temperature PR spectra of InGaN QWs with different In content (indicated). Arrows indicate the optical transition energies estimated by fitting the experimental curves (thin lines) with the Lorentzian functional form (bold lines).

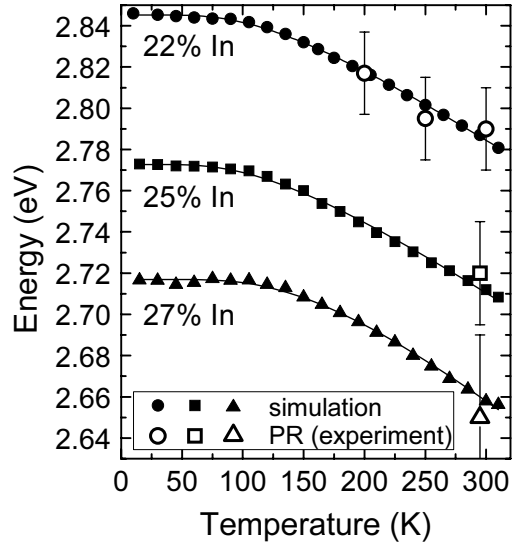

Fig. 5 Reconstructed average exciton energies (solid points) as a function of temperature for InGaN QWs with different In content (indicated). Solid lines represent the best fits by using a Bose-Einstein-like formula. Open points show the PR resonance energies.

The densities of band-tail-states used in the simulations with inhomogeneous broadening taken into account were collated with their respective PLE spectra for each sample (see thin lines in Fig. 3). The densities of band-tail-states were centered at the exciton energies (see arrows in Fig. 3) reconstructed by using the peak position of the simulated spectra and the measured peak positions of the PL bands at $77 \mathrm{~K}$. The coincidence between the long-wavelength slopes of the densities of band-tail-states and that of PLE spectra is found to be fairly good, what also supports the quantitative estimates of the band edge in the InGaN MQWs with different In content.

4 Conclusion A quantitative analysis of the PL linewidth in $\operatorname{In}_{x} \mathrm{Ga}_{1-x} \mathrm{~N} / \mathrm{GaN}$ MQWs with different indium content $(x \approx 0.22-0.27)$ was performed by using Monte Carlo simulation of exciton hopping. The simulation results imply that exciton motion in InGaN alloy can be universally described in terms of exciton hopping through localized states within relatively large In-rich regions dispersed in the average exciton energy. An increase of indium content was found to enlarge the band potential roughness within the In-rich regions as well as dispersion of average localization depth in these regions. The simulation results, which are in agreement with the PR and PLE data, enabled us to reconstruct the temperature dependence of the exciton energy.

Acknowledgements This research was partially supported by the joint Lithuanian-Latvian-Taiwan grant. A. Ž. and G. T. acknowledge the Lithuanian Ministry of Education and Science for their Fellowship.

\section{References}

[1] S. Chichibu, T. Sota, K. Wada, and S. Nakamura, J. Vac. Sci. Technol. B 16, 2204 (1998).

[2] S. Chichibu, K. Wada, and S. Nakamura, Appl. Phys. Lett. 71, 2346 (1997).

[3] A. Vertikov, A. V. Nurmikko, K. Doverspike, G. Bulman, and J. Edmond, Appl. Phys. Lett. 73, 493 (1998).

[4] Y. Narukawa, Y. Kawakami, M. Funato, S. Fujita, S. Fujita, and S. Nakamura, Appl. Phys. Lett. 70, 981 (1997).

[5] Y. S. Lin, K. J. Ma, C. Hsu, S. W. Feng, Y. C. Cheng, C. C. Liao, C. C. Yang, C. C. Chou, C. M. Lee, and J. I. Chyi, Appl. Phys. Lett. 77, 2988 (2000).

[6] K. Kazlauskas, G. Tamulaitis, A. Žukauskas, M. A. Khan, J. W. Yang, J. Zhang, G. Simin, M. S. Shur, and R. Gaska, Appl. Phys. Lett. 83, 3722 (2003).

[7] S. D. Baranovskii, R. Eichmann, and P. Thomas, Phys. Rev. B 58, 13081, (1998).

[8] L. Viña, S. Logothetidis, and M. Cardona, Phys. Rev. B 30, 1979 (1984). 